\section{miRNAs and malaria resistance}

The sickle cell hemoglobin variant (HbS) is known to confer resistance to the malaria parasite Plasmodium falciparum by various mechanisms that are not completely understood. Jen-Tsan Chi and colleagues investigated whether microRNAs (miRNAs) are involved in HbS-mediated malaria resistance and now report a novel miRNA-driven mechanism that contributes to malaria resistance in erythrocytes carrying the HbS variant (Cell Host Microbe 12, 187-199, 2012). The authors confirmed the presence of intact human miRNAs in the parasites and found enrichment of miR-451, miR-223 and miR-19b. They further showed that the miRNAs are transported into the parasite. The miRNAs are sufficient to confer resistance to wild-type HbAA red blood cells, and inhibition of miR-451 and miR-223 led to increased parasite growth in HbSS and $\mathrm{HbAS}$ red blood cells. P. falciparum does not encode Dicer/ Ago orthologs, so it was not clear how the human miRNAs affect parasite growth. Unexpectedly, the authors found that the human miRNAs are covalently bound to Plasmodium mRNAs, which appears to inhibit translation. The results suggest that ectopic introduction of miR-223 or miR-451 may be a potential therapeutic strategy for malaria.

\section{Single-stranded siRNA therapeutics}

Walt Lima and colleagues report the design of single-stranded small interfering RNAs (ss-siRNAs) that effectively silence targeted mRNAs in animals (Cell 150, 883-894, 2012). These ss-siRNAs have a modified, metabolically stable $5^{\prime}$ phosphate and can activate RNA interference (RNAi) in vitro and in mice. In the same issue, David Corey and colleagues, in collaboration with Walt Lima and colleagues, report the development of ss-siRNAs that can specifically inhibit a mutant huntingtin allele (Hdh) in mice (Cell 150, 895-908, 2012). Huntington disease is caused by the expansion of CAG trinucleotide repeats within the gene that encodes huntingtin (HTT). No effective drugs have been developed for this disease, and work to date has suggested that it may be effective to develop allele-specific inhibitors that only reduce mutant and not wild-type HTT. To this end, Corey and colleagues used systematic chemical modifications and iterative design to develop ss-siRNAs with ribose modifications and a $5^{\prime}$ end that is capped with (E)-vinyl phosphate. They found that an optimized ss-siRNA was capable of inhibiting mutant HTT in a fibroblast cell line derived from an individual with Huntington disease. They then showed that intraventricular delivery of a particular ss-siRNA could inhibit mutant HTT in a heterozygous mouse (Hdh $\left.{ }^{\mathrm{Q} 150 / \mathrm{Q} 7}\right)$ carrying a knock-in allele with 150 CAG repeats in exon 1.

PF

\section{New recessive autism syndrome}

Gaia Novarino and colleagues have identified mutations in the BCKDK gene as the cause of a potentially treatable recessive syndrome characterized by autism, intellectual disability and epilepsy (Science, published online 6 September 2012; doi:10.1126/science.1224631). The authors performed exome sequencing in two consanguineous families, each presenting with two siblings with autism and other neurodevelopmental phenotypes and a segregation pattern consistent with autosomal recessive inheritance. In both families, they found that the affected siblings harbored homozygous loss-of-function mutations in BCKDK. Further analyses of in-house exome data led to the identification of a third

Written by Orli Bahcall, Pamela Feliciano, Emily Niemitz \& Kyle Vogan consanguineous family with two affected siblings harboring a homozygous missense mutation in $B C K D K$. The kinase encoded by $B C K D K$ acts as a negative regulator of the branched-chain ketoacid dehydrogenase complex, which catalyzes the degradation of branched-chain amino acids. Consistent with the human findings, adult $B c k d k$-knockout mice developed neurological phenotypes, including tremors and seizures, accompanied by reduced levels of branched-chain amino acids in the brain and other tissues. Notably, placing these knockout mice on a diet enriched in branched-chain amino acids reversed these neurological phenotypes, suggesting that humans with $B C K D K$ mutations could benefit from similar dietary supplementation.

$K V$

\section{Transcription programs and cell division}

During cell division, mitotic chromosomes separate from the transcriptional machinery, silencing gene expression, but cell type-specific transcriptional programs persist through cell division and preserve lineage fidelity. Now, Gerd Blobel and colleagues show that the GATA1 transcription factor performs a 'bookmarking' function, transmitting hematopoietic transcriptional programs through mitosis (Cell 150, 725-737, 2012). The authors showed that GATA1 remains associated with mitotic chromatin in live erythroid cells, and they used chromatin immunoprecipitation with high-throughput sequencing to characterize the genome-wide occupancy of GATA1 in FACS-purified mitotic cells. This analysis showed retention of GATA1 occupancy during mitosis at some sites bound during interphase. Loci occupied by GATA1 in both interphase and mitosis (bookmarked loci) were enriched for genes that are essential for erythroid lineage differentiation. The authors further showed more rapid transcriptional reactivation of three target genes that remain occupied by GATA 1 during mitosis compared to three target genes that are occupied by GATA1 only during interphase. Finally, the authors devised a method to degrade GATA1 specifically during mitosis; this caused a slowing of transcriptional reactivation of GATA1-bookmarked genes. This work advances the understanding of the epigenetic mechanisms used to transmit gene expression programs through cell division.

$E N$

\section{Regulatory regions}

John Stamatoyannopoulos and colleagues report the enrichment of common disease-associated SNPs in regulatory DNA

(Science, published online 5 September 2012; doi:10.1126/ science.1222794). They performed genome-wide DNase I mapping in 349 cell and tissue samples from the ENCODE Project and the Roadmap Epigenomics Program. An average of 198,180 DNase I hypersensitive sites (DHSs) were identified per cell type, with $3,899,693$ distinct DHS positions identified in total. The authors compared these sites to noncoding GWAS-identified SNPs associated with 207 diseases and 447 quantitative traits, finding enrichment of these SNPs in DHSs, with $57.1 \%$ of all noncoding GWAS SNPs found within a DHS and a further $19.5 \%$ in complete linkage disequilibrium. This enrichment increased with both the strength and the extent of replication of the SNP-trait association. The authors also generated DNS maps from 233 fetal tissue samples and found that $88.1 \%$ of the noncoding SNPs were located within DHSs that are active in fetal cells, with $30.3 \%$ showing fetal stage-specific DHS activity that was not found in adult cells. The genic targets of 419 DHSs harboring disease-associated SNPs were identified, with many acting over a distance. The authors found that SNPs clustered in transcriptional regulatory pathways relevant to the disorder with which they were associated, with related diseases also showing a shared network. 